\title{
Large Scale Structure by Global Monopoles and Cold Dark Matter
}

\section{Leandros Perivolaropoulos}

\author{
Division of Theoretical Astrophysics \\ Harvard-Smithsonian Center for Astrophysics \\ 60 Garden St. \\ Cambridge, Mass. 02138 \\ and \\ Department of Physics \\ Brown University \\ Providence, R.I. 02912, U.S.A.
}

\begin{abstract}
A cosmological model in which the primordial perturbations are provided by global monopoles and in which the dark matter is cold has several interesting features. The model is normalized by choosing its single parameter within the bounds obtained from gravitational wave constraints and by demanding coherent velocity flows of about $600 \mathrm{~km} / \mathrm{sec}$ on scales of $50 h^{-1} \mathrm{Mpc}$. Using this normalization, the model predicts the existence of dominant structures with mass $2 \times 10^{16} M_{\odot}$ on a scale $35 h^{-1} M p c$ i.e. larger than the horizon at $t_{e q}$. The magnitude of the predicted mass function in the galactic mass range is in good agreement with the observed Schechter function.
\end{abstract}

\section{Introduction}

Recent observations have revealed the existence of nonlinear structures on very large scales (up to $50 h^{-1} \mathrm{Mpc}$ ). This observational fact has been a challenge for theoretical models during the last few years. In fact the currently 
popular models based on adiabatic primordial perturbations produced during inf1ation have difficulty to account for such large structures while maintaining successes on smaller scales. It is therefore important to investigate alternative theories.

A new class of such theories based on primordial perturbations produced by seeds rather than a superposition of waves with random phases has attracted significant attention during the past few years. Physically motivated candidates for such seeds are topological defects [1]. Topological defects are localized energy concentrations which are predicted by many particle physics models to form during phase transitions in the early universe. According to their geometry topological defects appear in the form of monopoles (stable pointlike defects), cosmic strings (linear defects) domain walls (planar defects) and textures (unstable pointlike defects). The vast majority of literature had until recently focused on gauged cosmic strings: linear defects interacting with short range forces. It was later realized[2] however that global defects interacting with long range forces, have two important advantages over gauged defects:

1. They generically have a larger correlation length during their evolution and can therefore seed structures on larger scales than gauged defects.

2. Their long range Goldstone field can provide efficient annihilation mechanisms that prevent them from dominating the energy density of the universe and lead to a scale invariant distribution: the scaling solution.

Thus, for example, monopole-antimonopole annihilation makes global monopoles consistent with standard cosmology [6], in contrast to the case of gauged monopoles. These advantages have led to recent extensive study of the unstable pointlike defect -global texture- with very encouraging results[河.

In this letter we consider a model in which the large scale structure is seeded by stable pointlike defects (global monopoles) and in which the dark matter is cold. Global monopoles may form in particle physics models where the breaking of a global symmetry results in a vacuum manifold with topology $S^{2}$. Such a breaking is realized in a model with the simple Langrangian density:

$$
\mathcal{L}=\frac{1}{2} \partial_{\mu} \vec{\Phi} \cdot \partial^{\mu} \vec{\Phi}-\frac{1}{4} \lambda\left(\vec{\Phi} \cdot \vec{\Phi}-\eta^{2}\right)^{2}
$$

where $\vec{\Phi}$ is a scalar field with $\mathrm{O}(3)$ global symmetry and the symmetry breaking is $O(3) \rightarrow O(2)$. Global monopoles are topologically nontrivial so- 
lutions of the above model. They are described by the spherically symmetric ansatz:

$$
\vec{\Phi}=f(r) \frac{\vec{r}}{r}
$$

Even though a solution for $f(r)$ is not known analytically, it is easy to show using the equations of motion that its asymptotic behaviour is $f(r) \rightarrow 1$ as $r \rightarrow \infty$ and $f(r) \rightarrow r$ for $r \rightarrow 0$. Thus the energy momentum tensor may be approximated at large distances by

$$
T_{t}^{t} \simeq T_{r}^{r} \simeq \frac{\eta^{2}}{r^{2}}, \quad T_{\theta}^{\theta}=T_{\phi}^{\phi} \simeq 0
$$

Solving the Einstein equations with this $T_{\nu}^{\mu}$ leads to the global monopole metric outside of the monopole core [4]

$$
d s^{2}=d t^{2}-d r^{2}-\left(1-8 \pi G \eta^{2}\right) r^{2}\left(d \theta^{2}+\sin ^{2} \theta d \phi^{2}\right)
$$

Thus there is a spherical deficit angle $\Delta=8 \pi G \eta^{2}$. Equivalently any planar surface containing the monopole, has the geometry of a cone with deficit angle $\Delta$. A more detailed analysis shows that in addition to the deficit angle the monopole induces an effective small negative mass $\mathrm{M}$ in its spacetime [11]. The magnitude of this mass however is proportional to the scale of symmetry breaking $\eta$ and is too small to be relevant for structure formation. On the other hand, the effects of the deficit angle are independent from the distance to the monopole core and may therefore be important even for very small $\Delta$. By obtaining the geodesic equations in the spacetime (4) it may be shown that a monopole moving with velocity $v_{m}$ induces a velocity perturbation $\Delta v$ towards the line of its trajectory, to its surrounding matter. Ignoring terms of $\mathrm{O}\left(\frac{v_{\text {matter }}}{v_{\text {monopole }}}\right)$ and $\mathrm{O}\left(\frac{v_{\text {matter }}}{c}\right)$, the magnitude of the dominant component of $\Delta v$ is $\Delta v=4 \pi G \eta^{2} v_{m} \gamma_{m}$ and its direction is perpendicular to the monopole trajectory . These primordial velocity perturbations may then grow to form galaxies, clusters and large scale structure. In what follows we obtain some of the predictions of the above described model. We make the assumption that the monopoles move with relativistic velocities on straight line trajectories. This assumption is justified due to the long range attractive monopole- antimonopole forces [四, [7] which are expected to induce relativistic velocities.

The structure of this paper is as follows: In the next section we use the Zeldovich approximation to obtain the growth of perturbations induced by global monopoles. Then we obtain the peculiar velocities predicted by the 
model and we use this result to normalize the single free parameter $\eta$ of the model. In section 3 we use the results of the Zeldovich approximation to obtain the spectrum of nonlinear masses predicted by the model.We also obtain the predicted mass function and we compare the galactic mass range with the observed Schechter function. Throughout the paper $h$ is the Hubble constant in units of $100 \mathrm{~km} /(\mathrm{sec} \cdot \mathrm{Mpc})$ and we consider a spatially f1at $\Omega=1$ universe.

\section{The Zeldovich Approximation}

We will use the Zeldovich approximation to calculate the growth of the velocity perturbations induced by relativistic global monopoles. The calculation is similar to the one in the case of cosmic string wakes [12], [8] with the important difference that in this case the geometry of each wake is cylindrical rather than planar. Consider a particle with unperturbed comoving distance $\mathrm{q}$ from the trajectory of a global monopole (assumed to be a straight line). Due to the initial velocity perturbation induced by the monopole a comoving position displacement $\Psi(q, t)$ will develop. Thus the physical distance $r_{\perp}$ from the monopole trajectory may be written

$$
r_{\perp}(q, t)=a(t)(q-\Psi(q, t))
$$

where $a(t)$ is the scale factor of the universe. Also, in the Newtonian approximation we have

$$
\begin{aligned}
\ddot{r}_{\perp} & =-\nabla r_{\perp} \Phi \\
\nabla_{r_{\perp}}^{2} \Phi & =4 \pi G \rho
\end{aligned}
$$

Using (5), (6), (7) and linearizing in $\Psi$ leads to

$$
\ddot{\Psi}+2 \frac{\dot{a}}{a} \dot{\Psi}+3 \frac{\ddot{a}}{a} \Psi=0
$$

The velocity perturbation induced by a monopole at time $t_{i}$ is $\Delta v=$ $4 \pi G v_{m} \gamma_{m}$. Therefore, the initial conditions to be used for the solution of (8) may be written in the comoving frame as:

$$
\begin{aligned}
& \Psi\left(t_{i}\right)=0, \dot{\Psi}\left(t_{i}\right)=\Delta v\left(\frac{t_{0}}{t_{i}}\right)^{\frac{2}{3}}, \quad t_{i}>t_{e q} \\
& \Psi\left(t_{i}\right)=0, \dot{\Psi}\left(t_{i}\right)=\Delta v\left(\frac{t_{0}}{t_{e q}}\right)^{\frac{2}{3}}\left(\frac{t_{e q}}{t_{i}}\right)^{\frac{1}{2}}, \quad t_{i}<t_{e q}
\end{aligned}
$$


The evolved comoving displcement $\Psi\left(t, t_{i}\right)$ with $t \geq t_{e q}$ is now easily obtained from (8), (9) and (10) and keeping only the growing mode it may be written as

$$
\begin{aligned}
\Psi_{>}\left(t, t_{i}\right) & =\frac{3}{5} \Delta v t_{0}^{\frac{2}{3}} t_{i}^{-\frac{1}{3}} t^{\frac{2}{3}}, \quad t_{i}>t_{e q} \\
\Psi_{<}\left(t, t_{i}\right) & =\frac{3}{5} \Delta v t_{0}^{\frac{2}{3}} t_{i}^{\frac{1}{2}} t^{\frac{2}{3}} t_{e q}^{-\frac{5}{6}}\left(1+\ln \left(\frac{t_{e q}}{t_{i}}\right)\right), \quad t_{i}<t_{e q}
\end{aligned}
$$

This result may be used to calculate several interesting quantities:

1. The large scale peculiar velocities predicted by the model.

2. The nonlinear mass of objects seeded at an initial time $t_{i}$.

3. The mass function (for this we will also need the scaling solution for monopoles).

Here we find the predicted peculiar velocities and use this result to normalize the single free parameter of the model $\eta$.

It is easy to show using (11) that the predicted peculiar velocity caused by a single monopole, coherent on a scale $q$ is

$$
v_{>}\left(t_{0}\right)=\dot{\Psi}_{>}\left(t_{0}\right)=\frac{2}{5}(\Delta v)\left(\frac{t_{0}}{t_{i}}\right)^{\frac{1}{3}}=\frac{60}{\nu^{\frac{1}{3}}}\left(G \eta^{2}\right)_{6} v_{m} \gamma_{m} q_{50}^{-1} \mathrm{~km} / \mathrm{sec}
$$

where $q_{50}$ is the comoving scale in units of $50 h^{-1} \mathrm{Mpc}, \nu \geq 1 \mid$ is the number of monopoles per horizon volume in the scaling solution $\left(t_{i}^{\text {com }}=\nu^{\frac{1}{3}} q\right)$ and $\left(G \eta^{2}\right)_{6}$ is $G \eta^{2}$ in units of $10^{-6}$. It may be shown using arguments similar to those in Ref. [5] that the effect of all later monopoles is to produce an rms velocity larger by a factor of 1.64 than the result (13). Typically $\nu^{\frac{1}{3}} \simeq 1[6]$. Demanding $\left.v_{>}\left(t_{0}\right)\right|_{q_{50}} \simeq 600 \mathrm{~km} / \mathrm{sec}$ we find $\left(G \eta^{2}\right)_{6} v_{m} \gamma_{m} \simeq 10$ implying, for $v_{m} \gamma_{m} \simeq 1$ a symmetry breaking scale $\eta$ for the monopole forming phase transition $\eta \simeq 3 \times 10^{16} \mathrm{GeV}$. This value of $\eta$ is consistent with the gravitational wave background constraints obtained in Ref.[13] $\eta \leq 5 \times 10^{16} \mathrm{GeV}$.

\section{$3 \quad$ Nonlinear Structures}

The solution $(11),(12)$ describes how the expansion of $r_{\perp}(t)$ is slowed down gravitationally due to the monopole induced velocity perturbation. The Zeldovich approximation used in deriving it can be used until the shell described by $r_{\perp}(t)$ turns around i.e. $\dot{r}_{\perp}(q, t)=0$ The condition $\dot{r}_{\perp}\left(q_{n l}, t\right)=0$ 
determines the thickness $q_{n l}$ of the cylindrical overdensity formed by the initial velocity perturbation. Using (5) it is easy to show that $q_{n l}\left(t, t_{i}\right)=$ $2 \Psi\left(t, t_{i}\right)$. The nonlinear scale $q_{n l}$ however can not grow beyond the comoving scale of the initial velocity perturbations $t_{i}^{\text {com }}$. Therefore, the correct expression for the turnaround scale at the present time $t_{0}$ is $q_{n l}\left(t_{0}, t_{i}\right)=$ $\min \left(t_{i}^{\mathrm{com}}, 2 \Psi\left(t_{0}, t_{i}\right)\right)$ or, defining $t_{*}^{\text {com }}=2 \Psi\left(t_{0}, t_{*}\right)$ we have:

$$
\begin{aligned}
& q_{n l}\left(t_{0}, t_{i}\right)=t_{i}^{\mathrm{com}}, \quad t_{i}<t_{*} \\
& q_{n l}\left(t_{0}, t_{i}\right)=2 \Psi\left(t_{0}, t_{i}\right), \quad t_{i}>t_{*}
\end{aligned}
$$

The form of $q_{n l}\left(t_{0}, t_{i}\right)$ is shown in Figure 1 for $t_{*}>t_{e q}$. Notice that for $t_{i} \leq t_{*}$ it is $t_{i}^{\text {com }}$, not $2 \Psi\left(t_{0}, t_{i}\right)$, that determines the scale $q_{n l}$. It is straightforward to calculate $t_{*}$ and show that $t_{*}=t_{e q} f(\lambda)$ where $\lambda=0.4\left(G \eta^{2}\right)_{6} h^{2} v_{m} \gamma_{m}$ and

$$
\begin{aligned}
& f(\lambda)=\mathrm{e}^{\frac{\lambda-1}{\lambda}}, \quad \lambda<1 \\
& f(\lambda)=\lambda^{\frac{3}{2}}, \quad \lambda>1
\end{aligned}
$$

In what follows we will use the normalization of the previous section and assume $\lambda=4 h^{2}>1$

The nonlinear mass corresponding to structure originating at time $t_{i}$ may be written as

$$
M\left(t, t_{i}\right)=\pi q_{n l}^{2}\left(t, t_{i}\right) v_{m} t_{i}^{\mathrm{com}} \bar{\rho}\left(t_{0}\right)
$$

For $\lambda>1$ we have $t_{*}>t_{e q}$ and using (14), (15) and (16) we obtain

$$
\begin{aligned}
M\left(t_{0}, t_{i}\right) & =\frac{v_{m}}{6 G}\left(\frac{t_{i}}{t_{e q}}\right)^{\frac{1}{2}} t_{i}, \quad t_{i}<t_{e q} \\
M\left(t_{0}, t_{i}\right) & =\frac{v_{m}}{6 G} t_{i}, \quad t_{e q}<t_{i}<t_{*} \\
M\left(t_{0}, t_{i}\right) & =\frac{6}{25} \frac{(\Delta v)^{2} v_{m}}{G}\left(\frac{t_{0}}{t_{i}}\right)^{\frac{1}{3}} t_{0}, \quad t_{i}>t_{*}
\end{aligned}
$$

For $t_{i}<t_{*}, q_{n l}\left(t_{0}, t_{i}\right) \simeq t_{i}^{\text {com }}$ and therefore all matter goes nonlinear on structures seeded before $t_{*}$. However, $\mathrm{M}\left(t_{0}, t_{i}\right)$ is an increasing function of $t_{i}$ and therefore structures seeded at $t_{i}<t_{*}$ can form by accreting structures seeded before $t_{i}$. On the other hand, for $t_{i}>t_{*}, \mathrm{M}\left(t_{0}, t_{i}\right)$ decreases with $t_{i}$ and since all matter has gone nonlinear in structures seeded before $t_{*}$, monopoles after $t_{*}$ can not produce nonlinear structures by today but can only induce large scale velocity f1ows. 
The important point to notice in Figure 1 is that the distinguished scale on which the largest and most prominent structures form is $t_{*}$ i.e. a scale which is larger than $t_{e q}^{\text {com }}$ by a factor $\lambda^{\frac{1}{2}}$. Thus the characteristic scale of dominant structures in the model is

$$
t_{*}^{\mathrm{com}}=\lambda^{\frac{1}{2}} t_{e q}^{\mathrm{com}}=35 h^{-1} M p c
$$

while the corresponding mass is

$$
M\left(t_{0}, t_{*}\right) \simeq 2 \times 10^{16} h^{-1} M_{\odot}
$$

where we have used the normalization obtained in the previous section $\lambda=$ $4 h^{2}$.

It is straightforward to now obtain the mass function predicted in the model using the additional input of the monopole scaling solution. Since the number of monopoles per Hubble volume per conformal horizon $\tau_{i}$ is $\frac{d n}{d \tau_{i}}=\frac{\nu}{\tau_{i}^{4}}$ with $\nu \simeq O(1)$, it is easy to see that the number density of nonlinear structures with mass larger than $\mathrm{M}$ is given by

$$
n_{>M}=\int_{M}^{M_{*}} d M \frac{\nu \frac{d \tau_{i}}{d M}}{\tau_{i}^{4}(M)}
$$

where $\mathrm{M}\left(t_{0}, t_{i}\right)$ was obtained in (17)-(19). It is straightforward to compute the integral (22) to find $n_{>M}$. The result is shown in Figure 2 and may be written as

$$
n_{>M}^{\text {model }}=\frac{\nu v_{m}}{144 G t_{0}^{2}}\left(M^{-1}-M_{*}^{-1}\right) \Theta\left(M_{*}-M\right)
$$

We also show the Schechter mass function (obtained from the Shechter luminocity function 10] with a mass to light ratio of $\left.100 \frac{M_{\odot}}{L \odot}\right)$ in Figure 2 for comparison with the monopole result. Clearly the model predicts a cutoff at the mass scale $M_{*}$. This cutoff however is much larger than the galactic mass and should be distinguished from the observed cutoff in the Schechter function. The Schechter function cuttof is expected to naturally emerge when effects of hydrodynamics are taken into account as was the case in Ref. [2]. In particular [14], cooling effects may provide the cutoff since the gas on larger scales can not cool in one Hubble time. As shown in Figure 2 the magnitude of the predicted mass function in the galactic mass range is in reasonably good agreement with the Schechter function given the assumptions involved in the calculation. The slope however is -1 for the monopole model but only $0.3 \pm 0.1$ for the observed Schechter function. This potential 
problem of the model could be resolved by considering hot dark matter or by introducing bias in the model.

We have therefore presented a study of a model for large scale structure formation based on primordial perturbations created by global monopoles and nonrelativistic dark matter (cold dark matter). The model has several encouraging features and clearly deserves further study. The model's single free parameter was fixed by demanding agreement with the observed large scale velocity flows. The obtained value was consistent with gravitational wave background bounds. The characteristic scale of dominant structures in the model is larger than the comoving horizon at $t_{e q}$ implying that the model has significant power on large scales. The main distinct feature of the monopole wakes considered here when compared to the cosmic string wakes is their generic cylindrical geometry to be compared with the planar geometry of string wakes. In addition, the global nature of the field in the monopole model implies the existence of larger correlation length in the scaling solution compared to the case of cosmic strings. Thus the parameters of the scaling solution in the case of monopoles favor the formation larger structures than cosmic strings.

One of the potential problems of the model is its prediction that all mass has gone nonlinear at the present time $t_{0}$. This problem, which also appears in the case of cosmic strings with cold dark matter could be resolved by considering relativistic particles as the dark matter (hot dark matter). Work in this direction is in progress. The consideration of hot dark matter could also soften the slope of the mass function which is predicted to be steeper than the Schechter function for galaxies.

The global monopole model for structure formation predicts a distinct signature on the microwave backgroud. In particular, a global monopole moving perpendicular to the line of sight of the observer will produce a temperature dipole in the microwave sky i.e. a hot-cold spot pair. The detailed form, magnitude and distribution of this signature will be presented in a separate publication [16].

\section{Acknowledgments:}

I would like to thank Robert Brandenberger and Tanmay Vachaspati for interesting conversations. This work was supported by a CfA Postdoctoral Fellowship.

\section{Figure Captions}

Figure 1: The nonlinear mass $q_{n l}$ vs time of initial perturbation $t_{i}$. 
Figure 2: The predicted mass function superposed with the observed Schechter function (obtained by using a fixed mass to light ratio).

\section{References}

[1] For a good review see A. Vilenkin, Phys. Rep.,121, 263 (1985).

[2] N. Turok, Phys. Rev. Lett., 63, 2625, (1989).

[3] A. Gooding, C. Park, D. Spergel, N. Turok, R. Gott, III, "The Formation of Structure in a Texture Seeded CDM cosmogony. " , Princeton preprint PUP-TH-1265 (1991).

[4] M. Barriola and A. Vilenkin, Phys. Rev. Lett., 63, 341 (1989).

[5] T. Vachaspati, "Distribution of Peculiar Velocities from Cosmic Strings", Tufts preprint (1991).

[6] S.H. Rhie and D.P. Bennett, Phys. Rev. Lett., 65 , 1709 (1990).

[7] L. Perivolaropoulos, "Instabilities and Interactions of Global Topological Defects". To appear in Nucl. Phys.B (1992).

[8] L. Perivolaropoulos, R. Brandenberger and A. Stebbins, Phys. Rev.,D41, 1744 (1990).

[9] E. Bertschinger, Ap. J.,316,489 (1987).

[10] G. Efstathiou and J. Silk: Fund. Cosmic Phys., 9,1 (1983).

[11] D. Harari and C. Lousto, Phys. Rev., D42, 2626 (1990).

[12] A. Stebbins,S. Veeraraghavan,R. Brandenberger,J. Silk and N. Turok, Ap. J.,322, 1 (1987).

[13] L. Krauss, "Gravitational Waves from Global Phase Transitions", Yale preprint YCTP-P33-91.

[14] M. J. Rees and J. P. Ostriker, MNRAS, 179, 541 (1977).

[15] L. Perivolaropoulos, "Nontopological Global Field Dynamics". Submitted to Phys. Rev.D (1991).

[16] L. Perivolaropoulos The MBR signature of Global Monopoles in preparation (1992). 